\title{
Mode-Selective Wavelength Conversion Based on Four-Wave Mixing in a Multimode Silicon Waveguide
}

Ding, Yunhong; Xu, Jing; Ou, Haiyan; Peucheret, Christophe

Published in:

OSA Technical Digest

Link to article, DOI:

10.1049/cp.2013.1339

Publication date:

2013

Link back to DTU Orbit

Citation (APA):

Ding, Y., Xu, J., Ou, H., \& Peucheret, C. (2013). Mode-Selective Wavelength Conversion Based on Four-Wave Mixing in a Multimode Silicon Waveguide. In OSA Technical Digest (pp. Tu.1.C.3). Optical Society of America. https://doi.org/10.1049/cp.2013.1339

\section{General rights}

Copyright and moral rights for the publications made accessible in the public portal are retained by the authors and/or other copyright owners and it is a condition of accessing publications that users recognise and abide by the legal requirements associated with these rights.

- Users may download and print one copy of any publication from the public portal for the purpose of private study or research.

- You may not further distribute the material or use it for any profit-making activity or commercial gain

- You may freely distribute the URL identifying the publication in the public portal 


\title{
Mode-Selective Wavelength Conversion Based on Four-Wave Mixing in a Multimode Silicon Waveguide
}

\author{
Yunhong Ding, Jing Xu, Haiyan Ou, and Christophe Peucheret \\ Department of Photonics Engineering, Technical University of Denmark, 2800 Kgs. Lyngby, Denmark \\ yudin@fotonik.dtu.dk
}

\begin{abstract}
We report all-optical mode-selective wavelength conversion based on four-wave mixing in a multimode Si waveguide. A two-mode division multiplexing circuit using tapered directional coupler based (de)multiplexers is used for the application. Experimental results show clear eye-diagrams and moderate power penalties for the conversion of both modes.
\end{abstract}

\section{Introduction}

Mode division multiplexing (MDM) has been successfully demonstrated as an efficient mean to increase the communication capacity of single fibers in telecommunication systems ${ }^{1}$. This technology is also promising in order to increase the capacity of silicon databusses for on-chip optical interconnections ${ }^{2-7}$. MDM may be used to enhance the throughput of the interconnections while limiting the number of required optical sources $^{2}$, whose integration onto the silicon platform is still the object of investigations. In wavelength division multiplexing (WDM) networks, wavelength-conversion is an essential functionality. Similarly, in MDM systems also exploiting the wavelength dimension, being able to perform wavelength conversion of the channels would offer new degrees of freedom for the implementation of both fibre and on-chip networks. In this context, mode-selective wavelength conversion is an important functionality, which has not been reported yet.

Here, we propose and demonstrate a novel all-optical (spatial) mode-selective wavelength conversion based on four-wave mixing (FWM) in a multimode silicon waveguide. A tapered directional coupler $(\mathrm{DC})$ based $\mathrm{TE}_{0} \& \mathrm{TE}_{1}$ mode multiplexer is utilized to couple two input channels to two spatial modes of the multimode silicon waveguide. By matching the spatial mode of the pump with that of the signal, idlers are generated from each channel on different modes, and consequently output to different demultiplexing ports. System experiments are carried out to demonstrate the concept using carrier-suppressed return-to-zero (CSRZ) signals at $40 \mathrm{Gbit} / \mathrm{s}$. The experimental results show clear eye diagrams and $1.3 \mathrm{~dB}$ and $2.8 \mathrm{~dB}$ power penalty for the conversion of each mode taken individually, as well as $2.4 \mathrm{~dB}$ and $4.9 \mathrm{~dB}$ excess conversion penalty when both signal modes co-propagate in the waveguide.

\section{Principle}

The principle of the on-chip mode-selective



Fig. 1: Principle of mode-selective wavelength conversion based on FWM. Two signal channels $\mathrm{CH}_{1}$ and $\mathrm{CH}_{2}$ are multiplexed to a multimode waveguide. Pump light is input from (a) port (1) and (b) port (2), generating idlers on mode 1 and 2 , respectively.

wavelength conversion is schematically shown in Fig. 1. Two signal channels $\mathrm{CH}_{1}$ and $\mathrm{CH}_{2}$ are multiplexed to a single multimode silicon waveguide on mode 1 and 2, respectively. If the pump light is input from the same port as $\mathrm{CH}_{1}$, it will be coupled to mode 1 in the multimode silicon waveguide, generating a strong idler from $\mathrm{CH}_{1}$ on the same mode by FWM. On the other hand, if the pump light is input from the same port as $\mathrm{CH}_{2}$, it will be coupled to mode 2 in the multimode waveguide, generating a strong idler from $\mathrm{CH}_{2}$ on that mode. The generated idlers will be output to different demultiplexing ports depending on their mode, where they can be spectrally filtered out and detected.

\section{Device fabrication and characterization}

In order to validate our proposal, an on-chip twomode division multiplexing circuit with $4 \mathrm{~mm}$ long straight multimode silicon waveguide ${ }^{4}$, as schematically shown in Fig. 2(a), was fabricated on a SOI wafer (top silicon layer: $250 \mathrm{~nm}$, buried silicon dioxide layer: $1 \mu \mathrm{m})$. Tapered DCs are used as $T E_{0} \& T E_{1}$ mode (de)multiplexers thanks to their simple structure and larger fabrication tolerance than normal $\mathrm{DCs}^{4,8}$. A single step of E-beam lithography and inductively coupled plasma reactive ion etching was used for the 
(a)

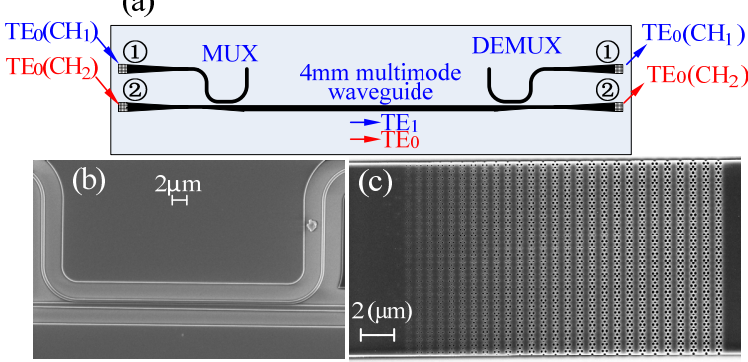

Fig. 2: (a) Schematic of the two-mode division multiplexing circuit. Scanning electron microscope (SEM) images of (b) a tapered DC based (de)multiplexer and (c) an apodized grating coupler.

fabrication. Signals fed to input ports (1) and (2), which consist of single-mode $\mathrm{TE}_{0}$ waveguides, are coupled to the $\mathrm{TE}_{1}$ and $T \mathrm{TE}_{0}$ modes in the multimode waveguide (waveguide width: $750 \mathrm{~nm}$ ), respectively, and output from different demultiplexing ports on the $\mathrm{TE}_{0}$ mode. In the tapered DC, the wide waveguide is tapered from $750 \mathrm{~nm}$ to $850 \mathrm{~nm}$ with tapering length of $30 \mu \mathrm{m}$ and coupling gap of $100 \mathrm{~nm}$, as shown in Fig. 2(b). In order to accommodate a high input light power, fully etched apodized grating couplers, which are based on photonic crystal structures, as shown in Fig. 2(c), were utilized to couple light to and from the chip. The total insertion losses are $11 \mathrm{~dB}$ and $14 \mathrm{~dB}$ between input/output (1)/(1) and (2)/(2), respectively, with mode crosstalk around $-15 \mathrm{~dB}$ and $-12 \mathrm{~dB}$ at $1550 \mathrm{~nm}$, as shown in Fig. 3. Note that the insertion losses include the coupling losses to standard single mode fibre (SSMF) of the grating couplers, as well as the insertion losses of the multiplexer and demultiplexer, and the propagation losses of the multimode waveguide. About $3 \mathrm{~dB}$ higher insertion loss is measured for $\mathrm{CH}_{1}$ because of the larger multiplexing loss and propagation loss of the $\mathrm{TE}_{1}$ mode compared to that of the $\mathrm{TE}_{0}$ mode.

\section{System experiment}

The fabricated chip was used to demonstrate mode-selective wavelength conversion with



Fig. 3: Measured transmission and mode crosstalk of the two channels $\left(\mathrm{CH}_{1}\right.$ and $\left.\mathrm{CH}_{2}\right)$ of the two-mode division multiplexing circuit.

CSRZ signals at $40 \mathrm{Gbit} / \mathrm{s}$. Fig. 4 illustrates the experimental setup. Pump light at wavelength $\lambda_{1}=1551.74 \mathrm{~nm}$ is modulated at $40 \mathrm{Gbit} / \mathrm{s}$ in the CSRZ format in two cascaded Mach-Zehnder modulators with a pseudo-random binary pattern length of $2^{31}-1$, and then amplified afterward by an erbium-doped fiber amplifier (EDFA). In our demonstration, modulation is imposed onto the pump in order to achieve a higher FWM conversion efficiency. The pump light is then split into two tributaries, each being amplified again by an EDFA and filtered out by an optical bandpass filter (OBPF) for noise suppression. A length of $1 \mathrm{~km}$ SSMF fiber is used to decorrelate the two pump tributaries. Polarization controllers (PCs) are introduced for each pump tributary to adjust its polarization to the TE mode of each input waveguide. Signal light at wavelength $\lambda_{2}=1554.47 \mathrm{~nm}$ is also amplified by an EDFA and split into two tributaries with a PC introduced for each tributary to excite the TE mode of the input waveguides. Each tributary of pump and signal light are combined by a $3 \mathrm{~dB}$ coupler and injected into the silicon chip for FWM. The generated idlers on the $T E_{0}$ and $T E_{1}$ modes are demultiplexed to different output ports and filtered out by an OBPF, and finally detected in a pre-amplified receiver.



Fig. 4: System experimental setup. The insets show the measured eye-diagrams of the CSRZ signals after the transmitter and that of the filtered idler from one of the outputs of the demultiplexer, respectively. 

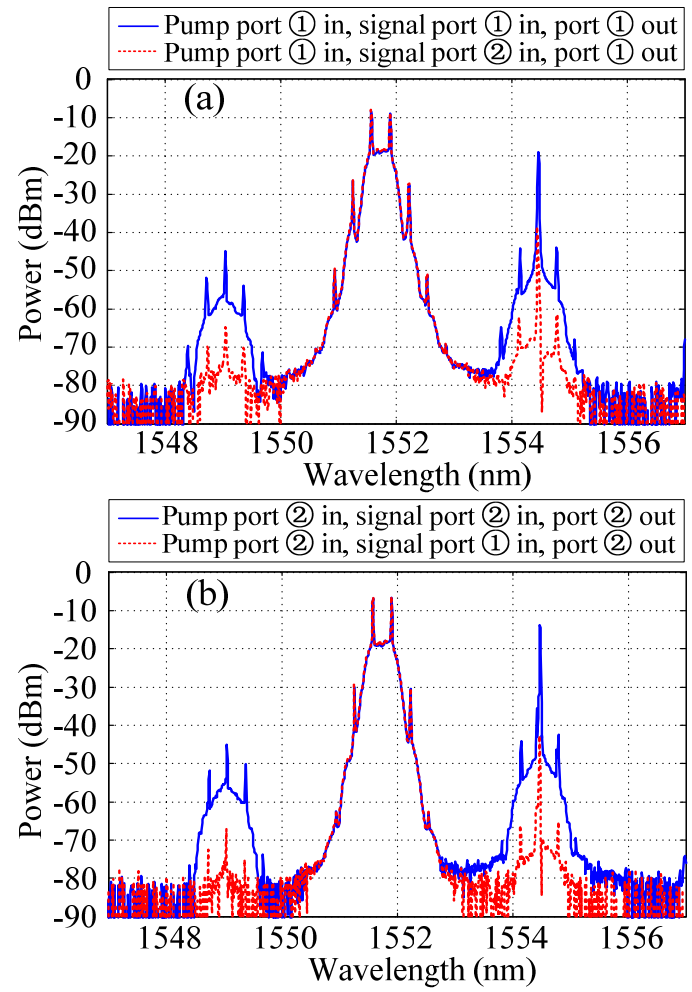

Fig. 5: Spectra measured at (a) ouput port (1) for pump input from (1), and signal light input from (1) or (2), respectively, and (b) output port (2) for pump input from (1), and signal light input from (1) or (2), respectively.

Fig. 5(a) and 5(b) show the measured FWM spectra at output ports (1) and (2), respectively, when the pump light is input from input ports (1) and (2), respectively, and the signal is input at either port (1) or port (2). Crosstalk induced by residual FWM (pump light is input from input port (1) and (2), signal light is input from input port (2) and (1), and detected at output port (1) and (2), respectively), which is caused by leakage light in the $T_{0} \& T E_{1}$ mode multiplexer, is also represented. Strong FWM is obtained when signal and pump lights are injected into the same multiplexing port. Meanwhile, very weak residual FWM is obtained if pump and signals are input from different multiplexing ports. The modal crosstalk on the idlers is better than $20 \mathrm{~dB}$ for both modes.

Fig. 6 shows the results of bit-error-ratio (BER) measurements performed for the two idlers obtained at output port (1) (corresponding to idler on the $\mathrm{TE}_{1}$ mode) or output port (2) (corresponding to idler on the $\mathrm{TE}_{0}$ mode) when pump and signal light are simultaneously input from input port (1) or input port (2), respectively (i.e. in the absence of modal crosstalk), as well as when signals are simultaneously input to ports (1) and (2) (i.e. in the presence of crosstalk). The corresponding eye-diagrams are also shown in the figure. Clear eye-diagrams are

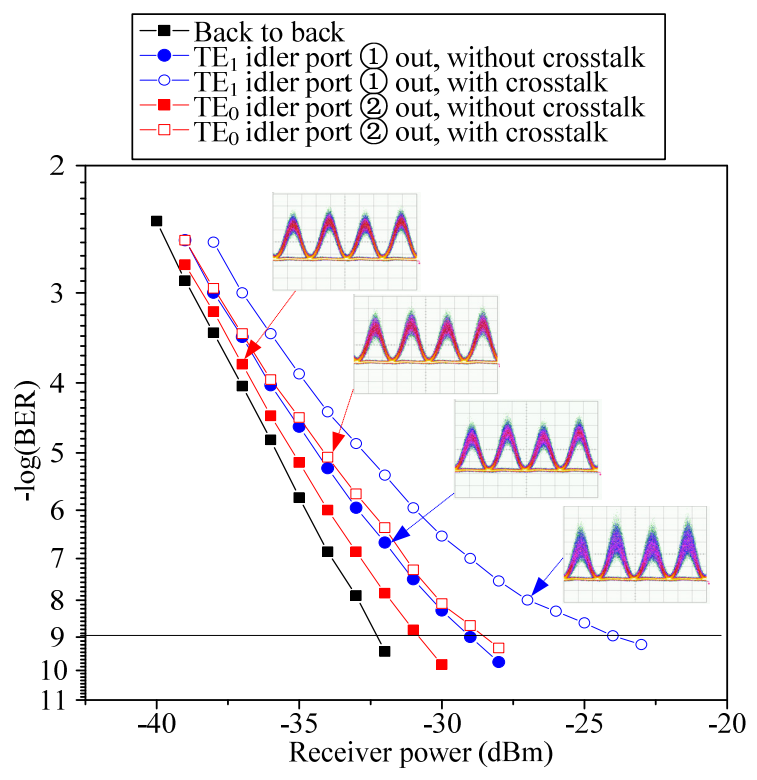

Fig. 6: $B E R$ measurement for the $T E_{0}$ and $T E_{1}$ idlers output from demultiplexing port (1) and (2), respectively, with and without crosstalk, and the corresponding eye-diagrams.

obtained for the idlers with and without crosstalk. In the absence of crosstalk, power penalties of $1.3 \mathrm{~dB}$ and $2.8 \mathrm{~dB}$ compared to the back-to-back case are obtained for the idlers output from port (1) and (2), respectively. An extra $2.4 \mathrm{~dB}$ and $4.9 \mathrm{~dB}$ power penalties are obtained with crosstalk, respectively.

\section{Conclusions}

We have successfully demonstrated on-chip mode-selective wavelength conversion based on FWM in a multimode silicon waveguide using a two-mode division multiplexing circuit. Modeselectivity is realized by launching pump light on different spatial modes. Experimental results show clear eye diagrams for conversion of the two modes with and without crosstalk and power penalties of $1.3 \mathrm{~dB}$ and $2.8 \mathrm{~dB}$ for the conversion of each mode taken individually, as well as $2.4 \mathrm{~dB}$ and $4.9 \mathrm{~dB}$ excess conversion penalty with crosstalk.

\section{References}

[1] R. Ryf et al., Proc. OFC'13, PDP5A.1 (2013).

[2] L. H. Gabrielli et al., Nature Commun. 3, 1271 (2012).

[3] D. Dai et al., Opt. Lett. 38, 1422 (2013).

[4] Y. Ding et al., Opt. Express 21, 10376 (2013).

[5] M. Greenberg and M. Orenstein, Opt. Express 13, 9381 (2005).

[6] S. Bagheri and W. M. J. Green, Proc. Group IV'09, 166 (2009).

[7] T. Uematsu et al., J. Lightwave Technol. 30, 2421 (2012).

[8] Y. Ding et al., Opt. Express 20, 20021 (2012). 